\title{
Cholesterol granuloma of the petrous apex: benefit of computer-aided surgery
}

\author{
Marco Caversaccio $\cdot$ Eugène Panosetti $\cdot$ \\ Panagiotis Ziglinas · Anton Lukes · Rudolf Häusler
}

Received: 7 December 2007 / Accepted: 21 May 2008 / Published online: 10 June 2008

(C) Springer-Verlag 2008

\begin{abstract}
The following is an analysis of the role of computer aided surgery by infralabyrinthine-subcochlear approach to the petrous apex for cholesterol granulomas with hearing preservation. In a retrospective case review from 1996 to 2008 six patients were analysed in our tertiary referral centre, otorhinolaryngology outpatient clinic. Excellent intraoperative localisation of the carotid artery, facial nerve and the entrance into the cholesterol cyst of the bone by means of the navigation system was seen. Additionally, the operation time decreased from an initial $4 \mathrm{~h}$ down to $2 \mathrm{~h}$. The application of computer-aided surgery allows intraoperative monitoring of the position of the tip of the microsurgical instruments in case of a rare disease and in the delicate area of the petrous apex giving a high security level.
\end{abstract}

Keywords Cholesterol granuloma $\cdot$ Petrous apex · Infralabyrinthine-subcochlear approach .

Navigation $\cdot$ Ear $\cdot$ CAS

M. Caversaccio $(\square) \cdot$ R. Häusler

Department of Otorhinolaryngology-Head and Neck Surgery, Inselspital University of Bern, Freiburgstrasse,

3010 Bern, Switzerland

e-mail: marco.caversaccio@insel.ch

E. Panosetti $\cdot$ P. Ziglinas

Département d'ORL, Centre hospitalier,

Luxembourg, Luxembourg

A. Lukes

Department of Neurosurgery,

Inselspital University of Bern, Bern, Switzerland

\section{Introduction}

Cholesterol granulomas (CG) (mucocele, cholesterol cyst) are rare and expansile, round or ovoid cysts containing cholesterol crystals surrounded by foreign body giant cells and chronic inflammation, all contained within a thick fibrous capsule [1]. Diagnosis of cholesterol granuloma of the petrous apex has become easier today with new imaging techniques such as MRI and CT [2]. The therapy of choice is surgery, which is still considered a delicate procedure. It is important to distinguish between CGs of the petrous apex and CGs that develop in the tympanomastoid compartment. The first one is supported more by the obstruction-vacuum theory, whilse the second is supported by sustained haemorrhage from the exposed marrow of the petrous apex [3]. One accepted surgical treatment of CGs is by way of ventilation tubes through a subcochlear or infralabyrinthine route when possible (Fig. 1). Both approaches have the advantage of hearing conservation. Occasionally, with far medial lesions, a transsphenoidal route of drainage is elected [1,4]. Although drainage procedures are often effective for a time, the ongoing secretion of bloody sludge often clogs the drainage tract. Recurrence on long-term follow-up ranges from 12 to $60 \%$ of cases [2, 5]. In recurrent cases, a reasonable strategy would be to separate the marrow compartment from the cyst cavity. This could be accomplished via middle cranial fossa approach, exposing and interrupting the cyst-marrow interface, e.g., bone wax.

Computer aided surgery (CAS) has become a common tool in skull base practice $[6,7]$. It is not substitute for anatomical knowledge under any circumstances. The main issue is accuracy and diminished time consumption which is influenced by the imaging modalities and registration methods [8]. Theoretically, the petrous bone should be the optimal substrate due to the absence of soft tissue shifting. 


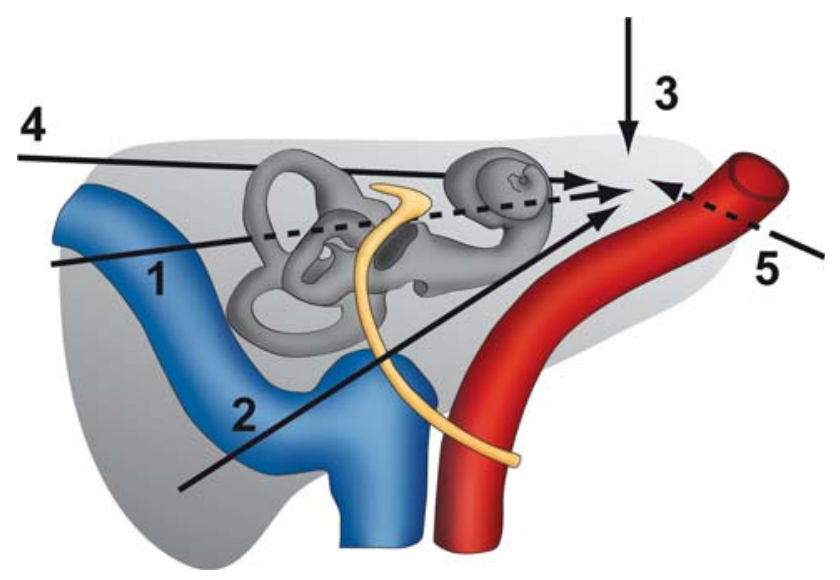

Fig. 1 The most important surgical approaches (red carotid artery, blue jugular bulb and sigmoid sinus, yellow facial nerve, grey dark labyrinthine and cochlea). 1 Transmastoidal-translabyrinthine, 2 transmastoidal infralabyrinthine-subcochlear, 3 transtemporal, 4 suboccipital, 5 transsphenoidal

The close relationship of the minute anatomical structures in the petrous bone demands a high degree of application accuracy of the image guidance system.

The objective is to demonstrate the advantage of a CAS system in CG drainage at the petrous apex.

\section{Patients and methods}

Between 1996 and 2008, six patients underwent surgery, five of them with CAS. In all six cases, the patients presented with unspecific vertigo, tinnitus, subjective hearing impairment and temporal headache lateralized toward the side of the petrous apex lesion. A pure tone audiogram was performed in all cases. Preoperative investigation was a combination of high-resolution CT and MRI. On the MRI, these erosive lesions are high intense in T1- and T2weighted sequences. Areas of interspersed low-signal intensity related to haemosiderin or granulation tissue are enhanced with contrast media. In the five subsequent cases, an infralabyrinthine/subcochlear approach was the method of choice. In the first case, a transmastoid-transtemporal approach was chosen. Additional cases where petrous apex cholesterin granulomas were discovered in MRI or CT were not treated by surgery because these were asymptomatic. The follow-up ranged between 6 months and 12 years.

The CAS protocol that we followed in our institution has been described in an earlier study by Caversaccio et al. [9] and was applied in each case. Pre-operative planning included helical computer tomography (CT) scan (General Electric, Milwaukee, WI, USA). A frameless optical system, the Surgigate ORL ${ }^{\text {TM }}$ (Medivision, CH-4436 Oberdorf) with a computer workstation (Sun Microsystems, Palo Alto, CA, USA) and a tracking system (Optotrak 3020, Northern Digital, ON, Canada) were used. The surgical instruments were equipped with four infrared emitting diodes. Prior to surgery, each surgical instrument was calibrated to verify its accuracy. Paired-point and surface matching were performed to compare the actual position of the skull with a pre-operative whole head CT scan (Fig. 2). An estimation of the anatomical verification was also carried out and known anatomical structures were evaluated for positional accuracy by measuring the deviation on multiples of the pixel size of the CT images in comparison to the clearly identified anatomical landmarks (between 0.3 and $0.6 \mathrm{~mm}){ }^{1}$

\section{Results}

All patients had CT or MR with well pneumatized mastoid air cells. The procedure duration for patients with cholesterol granuloma surgery at the beginning without navigation consisted of $7 \mathrm{~h}$ and the last two procedures were performed in $2 \mathrm{~h}$ (see Table 1). The mean preparation time for patients in whom CAS was applied was increased by

\footnotetext{
${ }^{1}$ Informed consent was obtained from every patient, in keeping with the mandate of the Declaration of Helsinki but the study was classified as exempt by the local institutional review board.
}

Fig. 2 Visible on the left side $\mathbf{a}$ in green are different points for surface matching as well in red anatomical landmarks for paired-point matching. On the right side $\mathbf{b}$ the surgeon with the navigated angled tool (small arrow) and the dynamic reference base (thick arrow) are visible
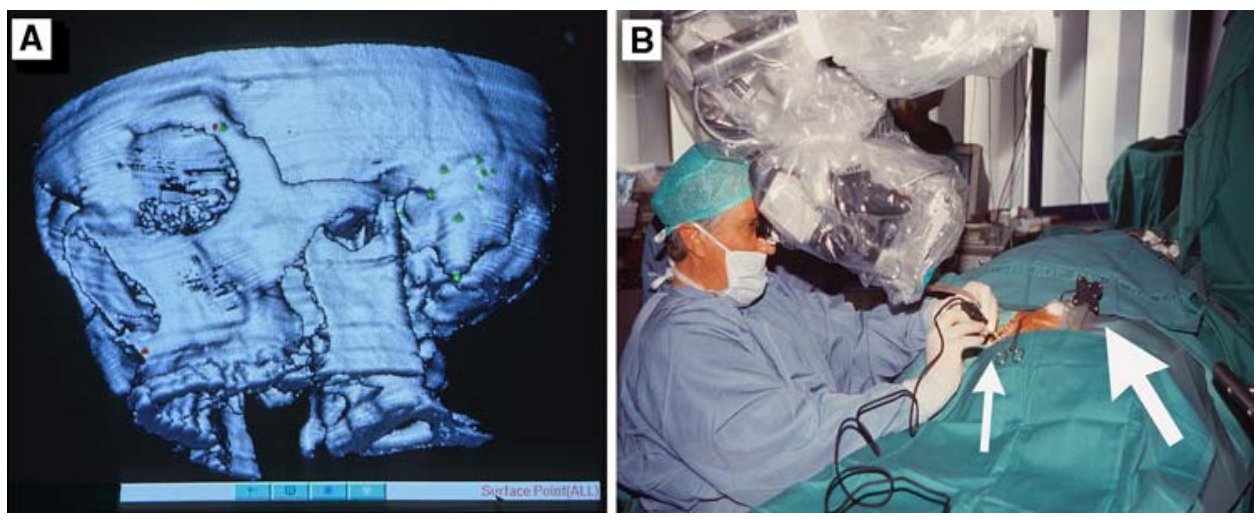
Table 1 Review of patients with cholesterol cysts of the temporal petrous apex treated with CAS (1996-2008) $n=6$

\begin{tabular}{llllllll}
\hline $\begin{array}{l}\text { Age } \\
(\mathrm{y})\end{array}$ & Sex & CT/MR & Surgical approach & CAS & $\begin{array}{l}\text { Operation } \\
\text { time }(\mathrm{h})\end{array}$ & Advantages & $\begin{array}{l}\text { Postop. hearing } \\
\text { threshold }(1 \mathrm{kHz})(\mathrm{dB})\end{array}$ \\
\hline 35 & $\mathrm{M}$ & Both & Transmastoid/transtemp. & & 7 & & 15 \\
$41^{\mathrm{a}}$ & $\mathrm{M}$ & Both & Infralab-subcochlear & $\mathrm{X}$ & 4 & Carotid artery, labyrinth & 35 \\
40 & $\mathrm{M}$ & Both & Infralabyrinthine & $\mathrm{X}$ & 2.5 & Carotid artery, labyrinth & 25 \\
$32^{\mathrm{b}}$ & $\mathrm{F}$ & Both & Infralabyrinthine & $\mathrm{X}$ & 2 & Carotid artery, labyrinth & 25 \\
35 & $\mathrm{M}$ & Both & Infralab-subcochlear & $\mathrm{X}$ & 2 & Carotid artery, labyrinth & 20 \\
45 & $\mathrm{~F}$ & Both & Infralabyrinthine & $\mathrm{X}$ & 2 & Carotid artery, labyrinth & 20 \\
\hline
\end{tabular}

${ }^{a}$ Middle ear revision

${ }^{\mathrm{b}}$ Recurrence of cholesterol granuloma

20 min. Navigation was used to plan the position and alignment of the localisation of the cholesterol granuloma, the facial nerve, the carotid artery, the labyrinth and the inner ear (Fig 3). This was done with ease using the navigated pointer and drill. In all procedures, the surgical landmarks were easily identified using CAS with a target accuracy range of $0.8-1.5 \mathrm{~mm}$. The accuracy depends on the manual registration technique under different conditions and on the point analysis in the plane ( $x$ and $y$ axis) or in the space ( $z$ axis). As the findings in the operative field can be correlated very well with CAS images, the surgeon has more confidence and less stress during the procedure (Fig. 4). There was ease in the handling of instruments equipped with IREDs and no major technical problem relating to CAS was noted in any of the procedures, except for one temporary software problem. The reconstruction of the middle ear was performed by incus interposition with good audiological output (Table 1). In one case, a recurrence with closure of the cyst wall occurred despite a drainage system into the Eustachian tube. During re-operation, a plugging with granulamtous tissue was seen. After cleaning and expanding the orifice, bicanalicular silicone tubes were inserted. There were no major complications such as facial palsy and no case of post-operative deafness.
Fig. 3 Intraoperative view (\#3) of the cholesterol granuloma (a). The crosshair indicates the tip of the ear pointer near the labyrinth; the postoperative result can be seen on the right side (b)
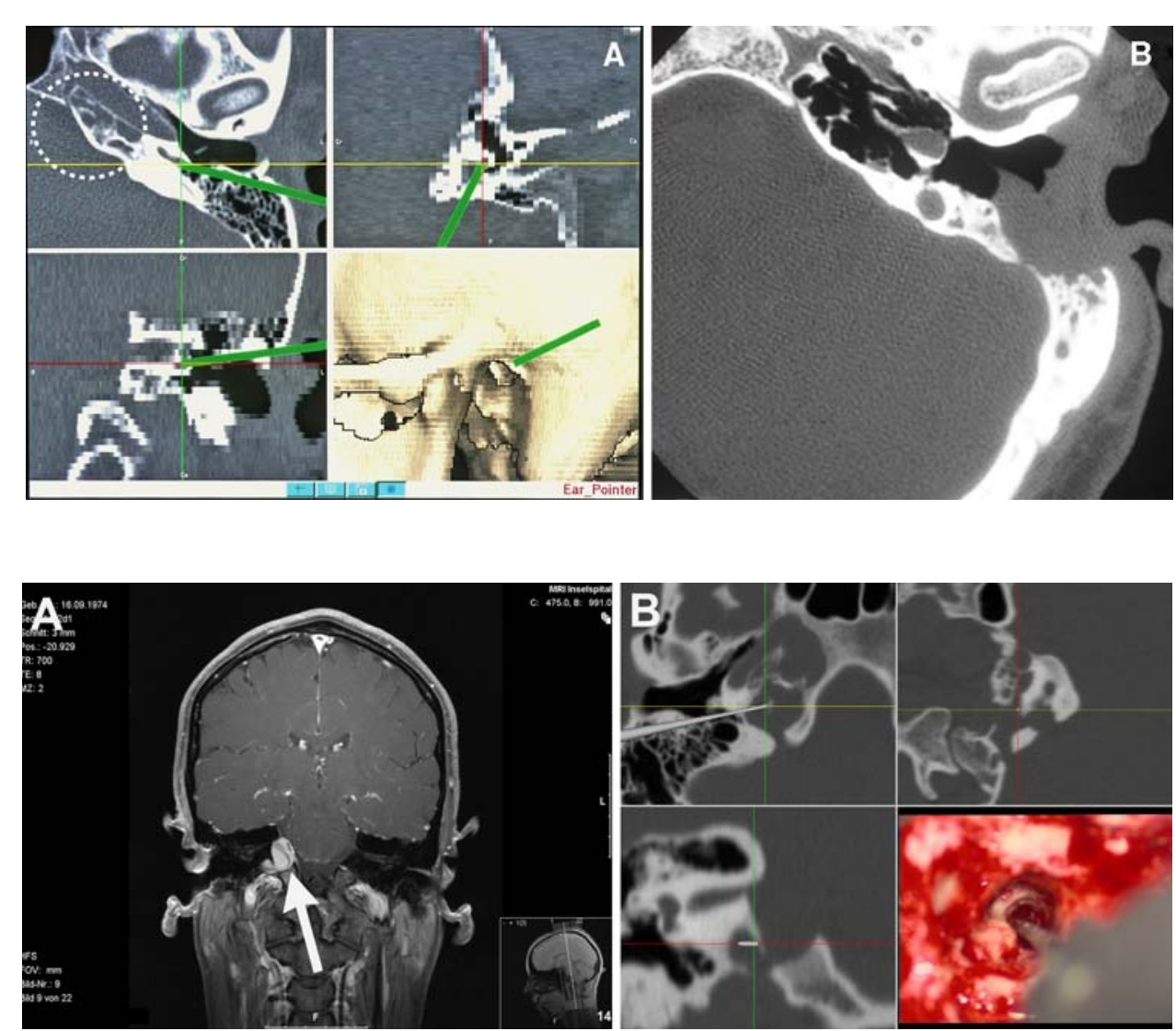

Fig. 4 Preoperative T2-weighted MRI with a cholesterol granuloma (\#4) on the right side (a) near the brain stem (arrow) with intraoperative view of an infralabyrinthine-subcochlear approach (b). The crosshair indicates the tip position inside the cholesterol granuloma cyst

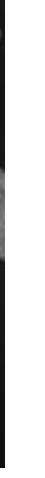




\section{Discussion}

CGs of the petrous apex is a rare disease. The petrous portion of the temporal bone lies in a complex anatomic position and has critical relationships to important neural and vascular structures. Consequently, lesions arising within or spreading to the petrous apex can result in severe clinical sequelae $[1,3]$. The treatment strategies of CGs of the petrous apex include drainage and/or complete excision of the capsule or procedures to drain the cyst. Complete surgical removal is rarely indicated because the lesion lacks an epithelial lining. Multiple surgical approaches have been described for treating these lesions. The choice of surgical approach depends on the location, extension of the lesion, anatomy of the surrounding structures, and the degree and quality of hearing. Our preferred approach is the infralabyrinthine-subcochlear method with complete mastoidectomy, canal wall down procedure, visualisation of the posterior semicircular canal, the carotid artery as well the jugular bulb.

The possibility of using image-guided surgery on the temporal bone has been discussed controversially for several years [10]. On one hand, there is a group of surgeons who have no possibility of buying a CAS system or they do not need it. On the other hand, there are proponents of this technique $[6,7]$. The advantage of the CAS system is the orientation in this complex area and, especially, the visualisation of the entrance to the cyst infralabyrinthine and the relationship to the carotid artery as well sinus sigmoideus. Additionally, the operation time was decreased, which could be also be supplementary result of the learning curve of the surgeon. This is a great advantage for the patient. An ossiculoplasty with incus interposition gives good clinical audiologic results; this is not possible in the case of a translabyrinthine approach. The disadvantage of solely using drainage is that often the sticky glue of granulomatous/haemosiderin tissue could, after several months, again block the drainage system (silicon-teflon- or gold tubes) in the middle ear and the Eustachian tube. We hope that CAS can support the surgeon in this very delicate area for the reasons of safety and quality, because the disease remains rare and, consequently, the surgeon's experience in the petrous apex stays limited.

\section{Conclusion}

The application of CAS for cholesterol granulomas on the petrous apex allows permanent intraoperative monitoring of the position of the microsurgical instruments (accuracy $<1.5 \mathrm{~mm}$ ). With this procedure, the operative time is decreased.

Acknowledgments The authors are supported by the Swiss National Research Foundation in the project: Computer Aided and Medical Interventions (http://www.co-me.ch)

\section{References}

1. Gacek RR (1993) Cystic lesions of the petrous apex. In: Nadol JB, Schuknecht HF (eds) Surgery of the ear and temporal bone. Raven Press, New York, pp 423-434

2. Thedinger BA, Nadol JB Jr, Montgomery WW, Thedinger BS, Greenberg JJ (1989) Radiographic diagnosis, surgical treatment, and long-term follow-up of cholesterol granulomas of the petrous apex. Laryngoscope 99:896-907

3. Jackler RK, Cho M (2003) A new theory to explain the genesis of petrous apex cholesterol granuloma. Otol Neurotol 24:96-106

4. Haberkamp TJ (1997) Surgical anatomy of the transtemporal approaches to the petrous apex. Am J Otol 18:501-506

5. Fong BP, Brackmann DE, Telischi FF (1995) The long-term follow-up of drainage procedures for petrous apex cholesterol granulomas. Arch Otolaryngol Head Neck Surg 121:426-430

6. Lenarz T, Heermann R (1999) Image-guided and computer-aided surgery in otology and neurotology: is there already a need for it? Am J Otol 20:143-144

7. Gunkel AR, Vogele M, Martin A, Bale RJ, Thumfart WF, Freysinger W (1999) Computer-aided surgery in the petrous bone. Laryngoscope 109:1793-1799

8. Van Havenbergh T, Koekelkoren E, De Ridder D, Van De Heyning P, Verlooy J (2003) Image guided surgery for petrous apex lesions. Acta Neurochir (Wien) 145:737-742

9. Caversaccio M, Zulliger D, Bächler R, Nolte LP, Häusler R (2000) Practical aspects for optimal registration (matching) on the lateral skull base with an optical frameless computer-aided pointer system. Am J Otol 21:863-870

10. Labadie RF, Majdani O, Fitzpatrick JM (2007) Image-guided technique in neurotology. Otolaryngol Clin North Am 40:611-624 